\title{
Multimodal All Optical Technique for the Quantification of Dynamic Stress
}

\author{
*Deptartment of Radiology, University of Southern California, MS \# 84650 Sunset Blvd., Los Angeles, \\ California, USA VSaxena@usc.edu
}

\begin{abstract}
A multimodal optical technique namely; near infrared diffuse optical spectroscopy (NIR: DOS) and mid infrared imaging (MIRI) is applied as a non-invasive, in-vivo tool for monitoring the vascular status of flexor pollicis brevis (thenar muscle of hand), and the physiological changes that occur during physical exercise. A technique helps in monitoring the changes in tissue blood oxygen saturation, and, information of the thermodynamics of heat dissipation during prolonged physical exercise. The proposed methodology is likely to provide insight into muscle physiology, therapeutic response and treatment.
\end{abstract}

\section{Introduction}

A new generation of excitation sources, detection technologies, and smart algorithms have renewed interest of using multi-spectral imaging techniques in the emerging field of biomedical diagnostics [1-3]. In this paper we present a multimodal optical technique based on steady state diffuse optical spectroscopy (SSDOS: 650-850 nm) for studying the changes in tissue blood oxygen saturation and mid infrared imaging (MIRI: 2-12 $\mu \mathrm{m}$ ) for studying the thermodynamics of heat dissipation by monitoring the changes in the spatial distribution of temperature during prolonged physical exercise. The understanding of tissue oxygenation status and the behaviour of the physiological parameters derived from thermometry may provide useful insight into muscle physiology, therapeutic response and treatment.

\section{Experimental Set-up and Protocol}

The NIRS system consists of a continuous wave (CW), steady state laser (650, 675, 780 and $850 \mathrm{~nm})$ diodes (2 mW each) as an excitation source, a probe, a high resolution spectrometer and a CCD detector optimized for the steady state diffuse optical spectroscopy (SSDOS) technique based on the theory of Farrell et al for monitoring the changes in tissue blood oxygen saturation [4]. The diffused signal was collected simultaneously in back reflection, spatially resolved geometry by arranging a probe that consists of a set of equidistant multimode fibers (100 $\mu \mathrm{m}$ core diameter, placed $2 \mathrm{~mm}$ apart with respect to each other) and $5 \mathrm{~mm}$ apart from the excitation fiber source in a planner geometry. An optical switch was employed to guide the diffused back-reflected light from individual fibers into a spectrometer and a high sensitivity (2 count/100 photons) CCD detector (Toshiba: QE65000, 1024 x 58 total pixels) interfaced with the computer for further data analysis. This method provides a straightforward and an accurate separation of absorption coefficient $\left(\mu_{\mathrm{a}}\right)$ and reduced scattering confident $\left(\mu_{\mathrm{s}}^{\prime}\right)$ from a single measurement from the spatially resolved diffuse reflectance [4]. Details of the theoretical model are described elsewhere [3,5]. A commercially available OXiMax N-600X model (Nellcor, USA) is used for measuring beats per minute (BPM). A ThermaCam infrared thermographic camera (S60-FLIR Systems, Boston) with a resolution of $1.3 \mathrm{mrad}$ and a sensitivity of $0.1^{\circ} \mathrm{C}$ at $30^{\circ} \mathrm{C}$ was used in our experiments. Detectors consisted of a micobolometer array of 320x 240 pixels and the captured images at a maximum speed of 3.4 frames per second were transferred to a laptop with an option to read out absolute temperature values directly from each pixel. The protocol is designed to study the physiology of the Flexor Pollicis Brevis (thenar muscles of right hand) during Isotonic exercise $(1 \mathrm{~Hz})$ and four minute rest. The studies were approved by institutional review board and 7 male Caucasian subjects (age range 28-35 years) were investigated under controlled environmental condition (Room temp: $65^{\circ} \mathrm{F}$, Humidity $60 \%$ ). The protocol consists of two components: NIRS and MIRI measurements. The NIRS investigation consisted of the measurement of oxy- and deoxy-hemoglobin to extract oxygen saturation values at the following stages. 1). Baseline measurements. 2). Isotonic exercise (flexing of thumb @1 Hz) measurements for one minute and during four minute rest. NIRS measurements were performed every 15-seconds (sampling time) by placing the probe head close to the Flexor Pollicis Brevis (thenar muscles of right hand) of all subjects (Figure 1).

\section{Results and Discussion}

The spatial distribution of temperature gradient on the skin surface is obtained by quantitative numerical analysis of several thermal images. The time sequential image analysis comprises of the following steps; first, the background (stationary temperature distribution) is subtracted from each frame. Secondly, the local and global region of interest 


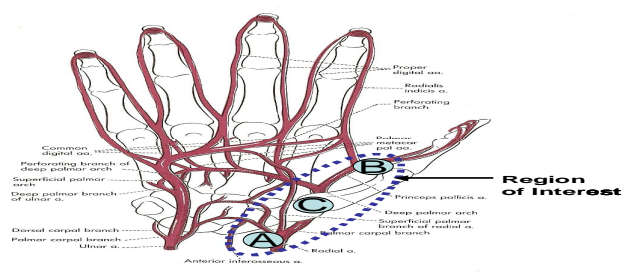

Figure 1. Region of interest of the flexor pollicis brevis.

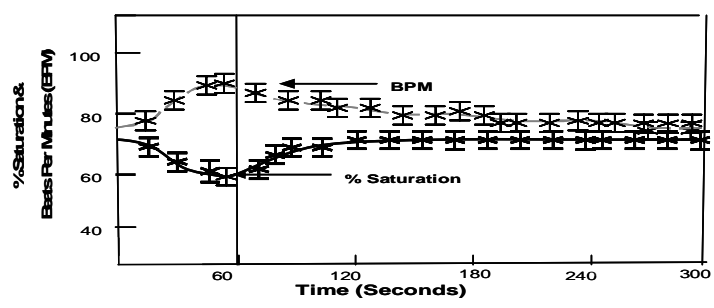

Fig. 2. Variation of oxygen saturation level and BPM.

(signatures) are defined and the spatial distribution of the temperature is plotted, and finally, the amplitude of temperature change with time is calculated. In the thermal images it is instructive in identifying hot and cold spots by enhancing areas of the image where temperature gradients are high or by measuring the asymmetries in the skin temperature distribution [3]. Figure (1) shows a typical region of interest shows a Point A (hot spot) corresponds to the position of radial artery as it enters the palm, before it bifurcates into the palmar carpal and the superficial palmar branch. Point C (cold spot) indicates the position where radial artery turns dorsally at the radial styloid process and Point B (hot spot) to the point where radial artery reemerges and bifurcates into proper digital arteries and radialis indicis artery. The temperature dynamics of the surface of palm at rest under thermo-neutral condition are determined mainly by peripheral blood flow, including that in arterioles and veins upto several millimeters deep. This blood flow provides a contrast demonstrating a significant temperature difference between vessels and surrounding tissues.

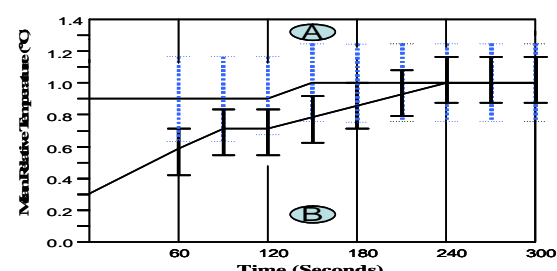

(a)

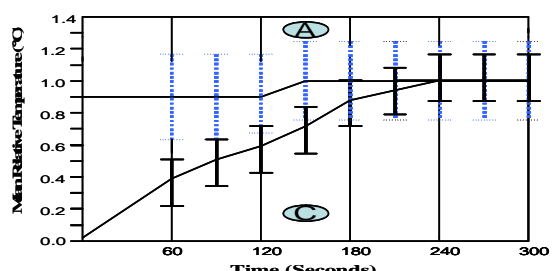

(b)

Fig. 3. Variation of mean relative temperature at the points of interest, (a): $A$ (upper curve) and $B$ (lowercurve), (b): $A$ (upper curve) and $C$ (lower curve) with time. After one minute of rigorous flexing of thumb, the mean relative temperature at point $B$ and $C$ rises to $0.3(0.3)^{\circ} \mathrm{C}$ and $0.4(0.2){ }^{\circ} \mathrm{C}$ respectively, while temperature at point $A$ changes slightly.

Figure $3(a, b)$ shows the variation of mean relative temperature at point $A, B$ and $C$ with time. The baseline temperature (mean standard deviation), before exercise at point $\mathrm{A}: 31.9(0.6)^{\circ} \mathrm{C}$ and $\mathrm{B}: 31.3(0.5)^{\circ} \mathrm{C}$ is higher than point C: $31.0(0.6)^{\circ} \mathrm{C}$. After one minute ofexercise, the mean relative temperature at point $\mathrm{B}$ and $\mathrm{C}$ rises to $0.3(0.3)^{\circ} \mathrm{C}$ and $0.4(0.2)^{\circ} \mathrm{C}$ respectively, while temperature at point $A$ slightly changes and the generated heat is removed by blood vessels via the process of convection. Thermal image taken after 240 seconds (4 minutes) clearly shows that the whole region of interest becomes worm and points $A, B$ and $C$ attain the same value of mean relative temperature. We simultaneously acquired oxygen saturation (NIRS) and beats per minute (BPM). It can be seen from figure (2), that after one minute of exercise, the maximum percentage change in oxygen saturation level $\left(\mathrm{SO}_{2}\right)$ is $14 \pm 5.0 \%$, within the first $50 \pm 5.0$ seconds from the onset of exercise. It is to be noted that at the same time interval, heart pumps more blood, as evident from the maximum percentage change in BPM $(\% \triangle B P M=13.0 \pm 5.0)$. After four minutes of rest, these values slowly recover towards their baseline. The examination of MIRI with the aid of computerized image processing, and pattern recognition techniques and NIR measurements enable the understanding of the distribution of skin temperature and oxygen saturation and is likely to help in understanding the muscle physiology, therapeutic response and treatment.

\section{REFERENCES}

[1] A. Bejan A., "From heat transfer principles to shape and structure in nature: Constructal Theory" Transactions of the ASME, Vol. 122, pp. 430-449, 2000.

[2] H.Schneider, Maier Tfleissner, J., Walther, J. M., Koidl, R., Weimann, G., Cabanski, W., Finck, M., Menger, P., Rode, W., Ziegler, J., "High-resolution 3-5 $\mu \mathrm{m} / 8-12 \mu \mathrm{m}$ m dual-band quantum well infrared photodetector array" Electron. Lett. Vol. 40, pp. 831-833, 2004.

[3] V. Saxena, and Nielsen, J. F., "An Integrated Bio-optical System for Oxygen Saturation Measurement" Physics of Medical Imaging, Proc. SPIE 5368, 29 San Jose (USA), 2004.

[4] T. Farrell, J., Patterson, M. S., and Wilson, B., "Time dependent blood flow and oxygenation in human skeletal muscles measured with noninvasive near infrared diffuse optical spectroscopy” Med. Phys. Vol. 19 (879), pp. 2449-2457, 1992.

[5] E. L. Hull, Nichols M. G., and Foster T. H., "Localization of luminescent inhomogeneities in turbid media with spatially resolved measurements of CW diffuse luminescence emittance” Applied Optics Vol. 37, pp. $13,1998$. 